PROCEEDINGS OF THE

AMERICAN MATHEMATICAL SOCIETY

Volume 135, Number 4, April 2007, Pages 1073-1080

S 0002-9939(06)08578-9

Article electronically published on September 26, 2006

\title{
HOW REGULAR CAN THE BOUNDARY OF A QUADRATIC SIEGEL DISK BE?
}

\author{
XAVIER BUFF AND ARNAUD CHÉRITAT \\ (Communicated by Linda Keen)
}

\begin{abstract}
In the family of quadratic polynomials with an irrationally indifferent fixed point, we show the existence of Siegel disks with a fine control on the degree of regularity of the linearizing map on their boundary. A general theorem is stated and proved. As a particular case, we show that in the quadratic family, there are Siegel disks whose boundaries are $C^{n}$ but not $C^{n+1}$ Jordan curves.
\end{abstract}

\section{INTRODUCTION}

In 1995, R. Pérez-Marco [PM1] constructed the first known examples of Siegel disks with $C^{\infty}$ boundaries. The authors $[\mathrm{BC}]$ discovered later an independent proof of the existence of Siegel disks with $C^{\infty}$ boundaries in the family of quadratic polynomials

$$
P_{\theta}(z)=\exp (i 2 \pi \theta) z+z^{2}, \quad \theta \in \mathbb{R} / \mathbb{Z} .
$$

A. Avila formalized, simplified and extended these results in $[\mathrm{A}]$ (these two preprints have now been published as $[\mathrm{ABC}]$ ). L. Geyer further simplified it in $\mathrm{G}]$.

These smooth Siegel disks were provided by an elementary construction based on a perturbation lemma (recalled as Lemma2 2 below), which was unknown at the time. Here, we describe a procedure which introduces irregularities on the boundaries of Siegel disks. For this, we use an old result of Herman (see Lemma 1 below). Combining it with our previous work, it enables us to prove much more: there exist quadratic Siegel disks for which the regularity of the boundary is anywhere in the full spectrum between analytic and continuous. Since the possibilities are numerous, we will formulate our main theorem in a general and abstract form. As a particular case, we will obtain that for all $n \in \mathbb{N}$, there exist quadratic Siegel disks whose boundaries are Jordan curves which are $C^{n}$ but not $C^{n+1}$ embedded curves.

To state the main theorem, let us introduce some notation. We will denote by $\mathbb{U}$ the set of complex numbers of norm 1 . If $P_{\theta}$ is linearizable in a neighborhood of its indifferent fixed point $z=0$, we denote by $\Delta_{\theta}$ its Siegel disk (maximal domain of linearization) and by $\phi_{\theta}: r_{\theta} \mathbb{D} \rightarrow \Delta_{\theta}$ the unique conformal map with $\phi_{\theta}(0)=0$ and $\phi_{\theta}^{\prime}(0)=1$. This $\phi_{\theta}$ is also the linearizing map and thus satisfies

$$
\phi_{\theta} \circ R_{\theta}=P_{\theta} \circ \phi_{\theta},
$$

Received by the editors January 28, 2005 and, in revised form, November 2, 2005.

2000 Mathematics Subject Classification. Primary 37F50, 37F10, 46B50.

(C)2006 American Mathematical Society Reverts to public domain 28 years from publication 
where $R_{\theta}(z)=\exp (i 2 \pi \theta) z$. If $P_{\theta}$ is not linearizable, we set $\Delta_{\theta}=\varnothing$ and $r_{\theta}=0$. Let $C^{0}$ be the space of holomorphic functions from $\mathbb{D}$ to $\mathbb{C}$ having a continuous extension to $\overline{\mathbb{D}}$. This is a Banach space for the supremum norm. Let $C^{\omega}$ be the space of functions from $\mathbb{D}$ to $\mathbb{C}$ having a holomorphic extension to a neighborhood of $\overline{\mathbb{D}}$.

Definition 1. Let $X$ and $Y$ be topological spaces and $X \subset Y$. We write $X \subset_{0} Y$ if the canonical injection $X \hookrightarrow Y$ is continuous. If moreover $X$ is a normed vector space and $Y$ a Fréchet space, we write $X \subset_{c} Y$ if every bounded set in $X$ has compact closure in $Y$.

Theorem 1. Let $F$ be any Fréchet space such that $C^{\omega} \subset F \subset_{0} C^{0}$, and let

$$
B \subset_{\mathrm{c}} F
$$

be a Banach space. Then, there exists a Bruno number $\theta$ such that

- the map $z \mapsto \phi_{\theta}\left(r_{\theta} z\right)$ belongs to $F$ but not to B;

- the boundary of $\Delta_{\theta}$ is a Jordan curve that does not contain the critical point of $P_{\theta}$.

Equivalently, one can replace the Banach space $B \subset_{c} F$ by a countable union of Banach spaces $B_{n} \subset_{c} F$ or by a countable union of compact sets $K_{n} \subset F$.

There are many Fréchet and Banach spaces or compact subsets that can be considered here. To give a sample of what can be obtained from Theorem 1, we will restrict ourselves to the following $C^{\alpha}$ conditions. Let $f: \mathbb{D} \rightarrow \mathbb{C}$ be holomorphic. If $\alpha=n$ is an integer, we say that $f \in C^{n}$ if $f^{(k)} \in C^{0}$ for $0 \leq k \leq n$. We say that $f \in C^{\infty}$ if $f \in C^{n}$ for every integer $n$. If $\alpha=n+\varepsilon$ for some integer $n$ and some $\varepsilon \in] 0,1\left[\right.$, we say that $f \in C^{n+\varepsilon}$ if $f \in C^{n}$ and $f^{(n)}$ is $\varepsilon$-Hölder on $\overline{\mathbb{D}}$. For $\alpha \in\left[0,+\infty\left[, C^{\alpha}\right.\right.$ is a Banach spact 1 and for $\alpha<\beta$, the injection of $C^{\beta}$ in $C^{\alpha}$ is compact (the proof is similar to the proof of Ascoli's theorem).

Geometry vs. Analysis. We control the regularity of the boundaries of Siegel disks via the regularity of the linearizing maps. We will need a few results on the boundary behavior of conformal maps for which we refer the reader to $\mathrm{Po}$. For $\alpha \geq 1$, there is a notion of $C^{\alpha}$ Jordan curve (see $[\mathrm{Po}$, section 3.3): these are curves that have a parameterization that is $C^{\alpha}$ with nonvanishing first derivative.

Theorem 2 (Kellogg-Warschawski). Assume $\alpha \in] 1,+\infty]$ is not an integer and $\Gamma \subset \mathbb{C}$ is a Jordan curve bounding a simply connected domain $U$ containing 0. Then, $\Gamma$ is a $C^{\alpha}$ Jordan curve if and only if the conformal map $\phi:(\mathbb{D}, 0) \rightarrow(U, 0)$ belongs to $C^{\alpha}$ with nonvanishing derivative on $\overline{\mathbb{D}}$.

The situation is less simple when $\alpha=n$ is a positive integer. If the conformal map of a Jordan curve $J$ is in $C^{n}$ and if the first derivative does not vanish, then obviously $J$ is a $C^{n}$ Jordan curve. However, there are $C^{n}$ Jordan curves whose conformal maps are not in the space $C^{n}$.

\footnotetext{
${ }^{1}$ For $\phi \in C^{n+\varepsilon}$ and $\left.\varepsilon \in\right] 0,1[$, we set $\|\phi\|_{C^{n+\varepsilon}}=\sum_{k=0}^{n}\left\|\phi^{(k)}\right\|_{\infty}+\min \left\{\lambda>0 \mid \lambda x^{\varepsilon}\right.$ is a modulus of continuity for $\left.\phi^{(n)}\right\}$.
} 
In the quadratic family, if the conformal map of a Siegel disk is at least $C^{1}$, then its derivative cannot vanish on $\overline{\mathbb{D}}$ (this is an easy exercise). Theorem 2 has the following consequence: for a noninteger $\alpha \in] 1,+\infty]$,

$$
z \mapsto \phi_{\theta}\left(r_{\theta} z\right) \in C^{\alpha} \Longleftrightarrow \partial \Delta_{\theta} \text { is a } C^{\alpha} \text { Jordan curve. }
$$

Let us now state some corollaries of Theorem 1 .

Corollary 1. There exist quadratic Siegel disks with $C^{\infty}$ boundaries.

Proof. Take $F=C^{\infty}$ and $K_{n}=\varnothing$.

In $[\mathrm{PZ}$, C. Petersen and S. Zakeri proved that for almost every $\theta \in \mathbb{R} / \mathbb{Z}$, the quadratic polynomial $P_{\theta}$ has a Siegel disk whose boundary contains the critical point, is a Jordan curve but is not a quasicircle. Theorem 1 implies:

Corollary 2. There exist quadratic Siegel disks whose boundaries are Jordan curves that do not contain the critical point and are not quasicircles.

Proof. The conformal map of a quasidisk is always Hölder (see $[\mathrm{Po}]$ ). So, choose $F=C^{0}$ and $B_{n}=C^{1 / n}(n \geq 2)$.

Corollary 3. For every $n \in \mathbb{N}$, there exist quadratic Siegel disks whose boundaries are Jordan curves that do not contain the critical point and are $C^{n}$ but not $C^{n+1}$ Jordan curves.

Proof. Take $F=C^{n}, B=C^{n+1 / 2}$. Then, Theorem 1 gives Siegel disks whose boundaries are $C^{n}$ but not $C^{n+1 / 2}$ Jordan curves. (Note that taking $B=C^{n+1}$ does not work; see the discussion Geometry vs. Analysis above.)

If $P_{\theta}$ has a Siegel disk, set

$$
I_{\theta}=\left\{\alpha \in \left[0,+\infty\left[\mid \phi_{\theta}\left(r_{\theta} z\right) \in C^{\alpha}\right\}\right.\right.
$$

This is an interval, either empty or with left bound $=0$. It contains 0 if and only if the boundary of $\Delta_{\theta}$ is a Jordan curve (see [M] or [Ro]). However, it is yet unknown if $\partial \Delta_{\theta}$ is always a Jordan curve. So, it is unknown if $I_{\theta}$ can be empty, but the following proves that all other cases happen.

Corollary 4. For every $\alpha \geq 0$, there exists a Bruno number $\theta$ such that $I_{\theta}=[0, \alpha]$ and for every $\beta>0$, there exists a Bruno number $\theta$ such that $I_{\theta}=[0, \beta[$.

Proof. For the first case, choose $F=C^{\alpha}$ and $B_{n}=C^{\alpha+1 / n}$. For the second case, choose $F=\bigcap_{\alpha \in[0, \beta[} C^{\alpha}, B=C^{\beta}$.

Remark 1. For $n \in \mathbb{N}$, let $C^{n+\text { Lip }}$ be the set of holomorphic functions on $\mathbb{D}$ whose $n$-th derivative is Lipschitz. The inclusion of $B=C^{n+1}$ in $F=C^{n+\text { Lip }}$ is not compact (it is an isometry). Thus we cannot apply Theorem 1. We may wonder whether there exist (fixed quadratic) Siegel disks whose conformal maps belong to $C^{n+\text { Lip }}$ but not $C^{n+1}$. In fact, for $n>0$, this is impossible by a theorem of Gottschalk and Hedlund (see [H1, Proposition 4.2 in part IV]). 


\section{Proof of Theorem 1}

It is equivalent to prove Theorem 1 for a Banach space $B \subset_{\mathrm{c}} F$, for a countable union of Banach spaces $B_{n} \subset_{c} F$ or for a countable union of compact sets $K_{n} \subset F$, as shown in the following

Proposition 1. If $F$ is a Fréchet space, then

(1) the image of any Banach space $B$ under a compact injection is contained in a countable union of compact subsets of $F$;

(2) any countable union of compact subsets $K_{n}$ of $F$ is contained in the image of a Banach space by a compact injection.

Proof. For (11), let $K_{n}$ be the closure in $F$ of the ball of radius $n$ in the $B$-norm. Obviously, $B \subset \bigcup_{n \in \mathbb{N}} K_{n}$. Moreover, by the very definition of a compact injection, $K_{n}$ is compact.

For (2), let us reduce the problem to the case of a single compact set. Let $\lambda_{n}>0$ be a sequence so that $\lambda_{n} K_{n} \longrightarrow 0$. Let $K=\{0\} \cup \bigcup_{n \in \mathbb{N}} \lambda_{n} K_{n}$. Then $K$ is compact, and any vector subspace of $F$ containing $K$ will contain $\bigcup_{n \in \mathbb{N}} K_{n}$. Next, we replace $K$ by $\overline{\mathbb{D}} K=\{\lambda f|| \lambda \mid \leq 1, f \in K\}$. Then we can replace $K$ by the closure of the convex hull of $K$, which is compact by a well-known property of compact sets in Fréchet spaces (see $[\mathrm{Ru}$, Theorem 3.20). Now $K$ is both convex and invariant under multiplication by elements of $\overline{\mathbb{D}}$. Now $B=\bigcup_{t \in \mathbb{R}_{+}} t K$ equipped with the norm $\|f\|_{B}=\inf \left\{t \in \mathbb{R}_{+} \mid f \in t K\right\}$ is a Banach space that is compactly injected in $F$.

We will prove Theorem 1 for a countable union of compact sets $K_{n} \subset F$. In fact, we will prove the following stronger version of Theorem 1 .

Theorem 3. Let $F$ be any Fréchet space such that

$$
C^{\omega} \subset F \subset_{0} C^{0}
$$

and let $\left(K_{n}\right)_{n \in \mathbb{N}}$ be any sequence of compact subsets of $F$. Then, for every Bruno number $\theta$, every $r<r_{\theta}$ and every $\varepsilon>0$, there exists a Bruno number $\theta^{\prime}$ such that

(1) $\left|\theta^{\prime}-\theta\right|<\varepsilon$;

(2) $r<r_{\theta^{\prime}}<r+\varepsilon$;

(3) the map $z \mapsto \phi_{\theta^{\prime}}\left(r_{\theta^{\prime}} z\right)$ belongs to $F$ but to no $K_{n}$;

(4) the distance in $F$ between $z \mapsto \phi_{\theta^{\prime}}\left(r_{\theta^{\prime}} z\right)$ and $z \mapsto \phi_{\theta}(r z)$ is $<\varepsilon$.

Moreover, the continuous extension $\psi$ of $\phi_{\theta^{\prime}}$ to $r_{\theta^{\prime}} \overline{\mathbb{D}}$, which exists by (3) , satisfies

(5) $\left\|\psi\left(r_{\theta^{\prime}} z\right)-\phi_{\theta}(r z)\right\|_{\infty}<\varepsilon$ on $\overline{\mathbb{D}}$;

(6) $\psi\left(r_{\theta^{\prime}} \mathbb{U}\right)=\partial \Delta_{\theta^{\prime}}$ is a Jordan curve that does not contain the critical point.

It is enough to prove (11)-(14) only. Indeed, (3) implies the existence of the extension since $F \subset C^{0}$. This extension must be injective (see [M], Lemma 18.7), which shows that the boundary of the Siegel disk is a Jordan curve. Then, (5) follows from (4) (with a smaller $\varepsilon$ ), by continuity of the inclusion $F \subset C^{0}$, and (5) implies that for $\varepsilon$ small enough, the boundary of the Siegel disk does not contain the critical point.

2.1. Tools. The proof is based on a perturbation Lemma 9, which will be stated in section 2.4. There are two results that make the perturbation lemma possible: Lemma 1 provides irregularity and Lemma 2 provides regularity. 
Let $\mathcal{D}_{2}$ denote the set of bounded type irrational numbers. For a proof of the following lemma, see for example [H2].

Lemma 1 (Herman). Let $\theta \in \mathcal{D}_{2}$, let $U$ be a connected open set containing 0 and let $f: U \rightarrow \mathbb{C}$ be a holomorphic function which fixes 0 with derivative $e^{2 \pi i \theta}$. Let $\Delta$ be the Siegel disk of $f$ at 0 (which exists by a theorem of Siegel). If $U$ is simply connected and $f$ is univalent, then $\Delta$ cannot have a compact closure in $U$.

Remark 2. In fact, Herman's theorem is stronger: $U$ need not be simply connected, and the condition on $\theta$ can be weakened to the so-called Herman-Yoccoz condition. See $[\mathrm{H} 2$ for a reference. In the case of quadratic polynomials, Lemma 1 is a consequence of the following fact: a bounded type quadratic Siegel disk contains the critical point on its boundary.

For a proof of the following lemma, see $\mathrm{BC}$ or $\mathrm{ABC}$.

Lemma 2. For every Bruno number $\theta$ and every $r<r_{\theta}$, there exists a sequence $\theta_{n} \in \mathcal{D}_{2}$ such that $\theta_{n} \longrightarrow \theta$ and $r_{\theta_{n}} \longrightarrow r$.

The references $\left[\mathrm{A}\right.$ and $\mathrm{G}$ ] contain similar statements without requiring $\theta_{n} \in \mathcal{D}_{2}$. It is in fact easy to adapt the proof in $\left[\mathrm{A}\right.$ and get $\mathcal{D}_{2}$ numbers. We do not know if G] can produce $\mathcal{D}_{2}$ numbers.

2.2. Elementary properties of the linearizations. Let us state well-known properties concerning the dependence of $\phi_{\theta}$ on $\theta$.

Lemma 3. Let $\theta_{n} \longrightarrow \theta$ be real numbers and assume that $r=\liminf r_{\theta_{n}}>0$. Then $r_{\theta} \geq r$ and $\phi_{\theta_{n}}$ tends to $\phi_{\theta}$ uniformly on compact subsets of $r \mathbb{D}$.

Proof. The univalent maps $\phi_{\theta_{n}}$ all have derivative 1 at 0 , hence form a normal family. It is therefore enough to prove that $\phi_{\theta}$ is the unique adherence value of the sequence $\phi_{\theta_{n}}$ on $B(0, r)$. Let $\psi$ be the limit of a convergent subsequence (for uniform convergence on compact subsets of $r \mathbb{D})$. Then $\psi^{\prime}(0)=1$, so in particular $\psi$ is not constant. Moreover, by passing to the limit in the equation $P_{\theta_{n}} \circ \phi_{\theta_{n}}=\phi_{\theta_{n}} \circ R_{\theta_{n}}$, we have $P_{\theta} \circ \psi=\psi \circ R_{\theta}$. Therefore, uniqueness of the linearization implies $r_{\theta} \geq r$ and $\psi=\phi_{\theta}$ on $r \mathbb{D}$.

Lemma 4. Assume $r_{n}>0, r_{n} \longrightarrow r>0, \theta \in \mathbb{R}$ and $\theta_{n} \longrightarrow \theta$ is a sequence of real numbers such that $r_{\theta_{n}}>r_{n}$, and the maps $z \mapsto \phi_{\theta_{n}}\left(r_{n} z\right)$ converge uniformly on $\mathbb{U}$ to some $\psi$. Then $r_{\theta} \geq r$, and $z \mapsto \psi(z)$ is a continuous extension of $z \mapsto \phi_{\theta}(r z)$ to the closed unit disk (if $r_{\theta}>r$, this just means $\phi_{\theta}(r z)=\psi(z)$ ). Moreover, $\psi$ is injective. In the case $r_{\theta}=r$, we have $\partial \Delta_{\theta}=\psi(\mathbb{U})$.

2.3. Preparatory work. Note that $C^{\omega}$ is not a Fréchet space. It is the union over $\varepsilon>0$ of spaces $C_{\varepsilon}^{\omega}$ of holomorphic functions on $(1+\varepsilon) \mathbb{D}$. Each $C_{\varepsilon}^{\omega}$ is endowed with the topology of uniform convergence on compact sets (in fact it is a Fréchet space; if you prefer Banach spaces, take the space of bounded holomorphic functions on $(1+\varepsilon) \mathbb{D}$ and the discussion below will be the same). We do not put a topology on $C^{\omega}$.

Lemma 5. Let $F \subset_{0} C^{0}$ be a Fréchet space and $K$ be a compact subset of $F$. Assume $f_{n} \in K$ tend to some map $f: \mathbb{D} \rightarrow \mathbb{C}$ uniformly on compact subsets of $\mathbb{D}$. Then $f \in K$ and

a) $d_{F}\left(f_{n}, f\right) \longrightarrow 0$ where $d_{F}$ is the distance function of the Fréchet space $F$,

b) $\left\|f_{n}-f\right\|_{\infty} \longrightarrow 0$. 
Proof. Part b) is a corollary of part a) and the continuity of the injection $F \subset C^{0}$. Since $f_{n}$ lies in the compact set $K$, it has a subsequence $f_{n_{k}}$ such that $d_{F}\left(f_{n_{k}}, h\right) \longrightarrow$ 0 for some $h \in K$. The injection $F \subset C^{0}$ being continuous, $\left\|f_{n_{k}}-h\right\|_{\infty} \longrightarrow 0$. Since $f_{n}$ tends to $f$ uniformly on compact subsets of $\mathbb{D}$, we must have $h=f$. This proves $f \in K$, and that $f$ is the only limit of convergent subsequences of $f_{n}$ in the $d_{F}$-metric. Since $f_{n}$ lies in a compact set, this gives part a).

Lemma 6. Assume $F$ is a Fréchet space with $C^{\omega} \subset F \subset_{0} C^{0}$. Then, for all $\varepsilon$, the injection $C_{\varepsilon}^{\omega} \subset F$ is continuous.

Proof. This is a corollary of the closed graph theorem, since the injection of $C_{\varepsilon}^{\omega}$ in $C^{0}$ is continuous.

Lemma 7. There exist subsets $D_{n}$ of $C^{\omega}$ with $C^{\omega}=\bigcup D_{n}$ such that for every Fréchet space $F$ with $C^{\omega} \subset F \subset_{0} C^{0}, D_{n}$ is compact in $F$.

Proof. Let $D_{n}$ be the set of holomorphic functions in $\left(1+\frac{1}{n+1}\right) \mathbb{D}$ whose absolute value is bounded by $n$. Each $D_{n}$ is compact in $C_{\frac{1}{n+1}}^{\omega}$ (Montel's theorem). According to Lemma 6. $D_{n}$ is also compact in $F$.

We will consider the following property of a subset $A$ of $C^{0}$ :

For every $\varepsilon>0, A$ contains a neighborhood of 0 in $C_{\varepsilon}^{\omega}$.

Lemma 8. For every Fréchet space $F$ with $C^{\omega} \subset F \subset_{0} C^{0}$, there exists a compact subset $L$ of $F$ with property $\mathcal{H}$.

Proof. Consider the $D_{n}$ from the proof of Lemma 7 . Since $D_{n}$ is compact in $F$, $\lambda D_{n}$ tends to 0 when $\lambda \longrightarrow 0$. Choose $\lambda_{n}>0$ such that $\lambda_{n} D_{n}$ is included in the ball of $F$ of center 0 and radius $1 /(n+1)$. Then the set $L=\bigcup \lambda_{n} D_{n}$ is compact (note that $0 \in L$ ) and has the property $\mathcal{H}$ since $D_{n}$ contains a neighborhood of 0 in $C_{\frac{1}{n}}^{\omega}$.

\subsection{Main lemma.}

Lemma 9 (Perturbation). Assume that $F$ is a Fréchet space with $C^{\omega} \subset F \subset_{0} C^{0}$, and $K$ is a compact subset of $F$. For every Bruno number $\theta$, every $\rho_{1}<\rho_{2}<r_{\theta}$, and every $\varepsilon>0$ there exists a Bruno number $\theta^{\prime}$ and $\rho^{\prime}>0$ such that

(1) $\left|\theta^{\prime}-\theta\right|<\varepsilon$,

(2) $\rho_{1}<\rho^{\prime}<\rho_{2}$,

(3) $r_{\theta^{\prime}}>\rho^{\prime}$,

(4) $d_{F}\left(\phi_{\theta}\left(\rho^{\prime} z\right), \phi_{\theta^{\prime}}\left(\rho^{\prime} z\right)\right)<\varepsilon$,

(5) $\phi_{\theta^{\prime}}\left(\rho^{\prime} z\right)$ does not belong to $K$.

Proof. Let $\rho_{3}=\frac{\rho_{1}+\rho_{2}}{2}$. If, for some $\left.\rho^{\prime} \in\right] \rho_{1}, \rho_{3}\left[, \phi_{\theta}\left(\rho^{\prime} z\right)\right.$ satisfies (5), then we are done with $\theta^{\prime}=\theta$.

Otherwise, use Lemma 2 to find $\theta_{n} \longrightarrow \theta$ such that $r_{\theta_{n}} \longrightarrow \rho_{3}$. By Lemma 3 , $\phi_{\theta_{n}}$ tends to $\phi_{\theta}$ uniformly on compact subsets of $\rho_{3} \mathbb{D}$. According to Lemma 6 , the injection $C_{\varepsilon}^{\omega} \subset F$ is continuous for all $\varepsilon>0$. On the other hand, there is some $\varepsilon>0$ such that the function $\left[\rho_{1}, \rho_{3}\right] \rightarrow C_{\varepsilon}^{\omega}$ defined by $\rho \mapsto \phi_{\theta}(\rho z)$ is continuous. Therefore, its image $K_{0}$ is compact in $C_{\varepsilon}^{\omega}$, hence compact in $F$.

Let $L$ be a compact subset of $F$ with property $\mathcal{H}$ as given by Lemma 8 . Set $K^{\prime}=(K+L) \cup\left(K_{0}+L\right)$. We claim that for large enough $n$, there exists a $\rho \in\left[\rho_{1}, r_{\theta_{n}}[\right.$ such that $\phi_{\theta_{n}}(\rho z)$ does not belong to $K^{\prime}$. Otherwise, by Lemma $5, \phi_{\theta_{n}}\left(r_{\theta_{n}} z\right)$ would 
belong to $K^{\prime}$. Lemma 5 b) would imply that $\left\|\phi_{\theta_{n}}\left(r_{\theta_{n}} z\right)-\phi_{\theta}\left(\rho_{3} z\right)\right\|_{\infty} \longrightarrow 0$. Thus eventually, $\partial \Delta_{\theta_{n}}$ would be contained in $\Delta_{\theta}$, contradicting Lemma 1 since $\theta_{n} \in \mathcal{D}_{2}$.

Then let $\rho_{n}^{\prime}$ be the infimum of the set of $\rho \in\left[\rho_{1}, r_{\theta_{n}}\left[\right.\right.$ such that $\phi_{\theta_{n}}(\rho z)$ does not belong to $K^{\prime}$. Note that $\phi_{\theta_{n}}\left(\rho_{n}^{\prime} z\right)$ does not belong to $K$. Otherwise, since $L$ has property $\mathcal{H}, \phi_{\theta_{n}}(\rho z)$ would belong to $K+L \subset K^{\prime}$ for all $\rho>\rho_{n}^{\prime}$ sufficiently close to $\rho_{n}^{\prime}$, which contradicts the definition of $\rho_{n}^{\prime}$. This gives (5) with $\rho^{\prime}=\rho_{n}^{\prime}$ for $n$ large enough.

Let us prove that $\rho_{n}^{\prime} \longrightarrow \rho_{3}$. Otherwise, for a subsequence, we would have $\rho_{n}^{\prime} \longrightarrow r \in\left[\rho_{1}, \rho_{3}\left[\right.\right.$. With the definition of $\rho_{n}^{\prime}$, this would yield a sequence $\rho_{n}^{\prime \prime} \longrightarrow r$ such that $\phi_{\theta_{n}}\left(\rho_{n}^{\prime \prime} z\right)$ does not belong to $K^{\prime}$. The sequence of holomorphic functions $z \mapsto \phi_{\theta_{n}}\left(\rho_{n}^{\prime \prime} z\right)$ would converge uniformly on compact subsets of $\frac{\rho_{3}}{r} \mathbb{D}$ to $\phi_{\theta}(r z)$. Because of property $\mathcal{H}$, the function $\left(\phi_{\theta_{n}}-\phi_{\theta}\right)\left(\rho_{n}^{\prime \prime} z\right)$ would eventually belong to $L$, so $\phi_{\theta_{n}}\left(\rho_{n}^{\prime \prime} z\right)$ would belong to $L+K_{0} \subset K^{\prime}$, which is a contradiction.

As soon as $\rho_{n}^{\prime}>\rho_{1}, \phi_{\theta_{n}}\left(\rho_{n}^{\prime} z\right)$ is in $K^{\prime}$ : indeed, for all $\rho \in\left[\rho_{1}, \rho_{n}^{\prime}\left[, \phi_{\theta_{n}}(\rho z)\right.\right.$ is in $K^{\prime}$, and the claim follows from Lemma 5 .

Lemma 5a) now implies that $d_{F}\left(\phi_{\theta_{n}}\left(\rho_{n}^{\prime} z\right), \phi_{\theta}\left(\rho_{3} z\right)\right) \longrightarrow 0$ when $n \longrightarrow+\infty$, and $\rho_{n}^{\prime} \longrightarrow \rho_{3}$ implies $d_{F}\left(\phi_{\theta}\left(\rho_{3} z\right), \phi_{\theta}\left(\rho_{n}^{\prime} z\right)\right) \longrightarrow 0$. This gives (44).

2.5. Proof of Theorem 3 , Let $D_{n}$ be given by Lemma 7 and $L$ by Lemma 8

We are going to define by induction on $n \geq-1$ a sequence $\theta_{n}$ of parameters, an increasing sequence $\rho_{n}^{\prime} \geq r$, and real numbers $\varepsilon_{n}>0$.

The induction hypothesis will be $H_{n}$ :

- $r_{\theta_{n}}>\rho_{n}^{\prime}$

- for all $k$ with $0 \leq k \leq n$, the $F$-distance between $\phi_{\theta_{n}}\left(\rho_{n}^{\prime} z\right)$ and the set $K_{k} \cup D_{k} \cup L$ is $>\varepsilon_{k}$.

Let $\theta_{-1}=\theta, \rho_{-1}^{\prime}=r, \varepsilon_{-1}=1$ ( $\varepsilon_{-1}$ will not be used).

Given $n \geq 0$, assume that $\theta_{k}, \rho_{k}^{\prime}, \varepsilon_{k}$ are defined for $-1 \leq k<n$, and that $H_{n-1}$ holds. There exists an $\eta>0$ such that for all $f \in F$ and all $k$ with $0 \leq k<n$, the condition $d_{F}\left(f, \phi_{\theta_{n-1}}\left(\rho_{n-1}^{\prime} z\right)\right)<\eta$ implies the $F$-distance between $f$ and $K_{k} \cup D_{k} \cup L$ remains $>\varepsilon_{k}$. Let $\rho_{1}=\rho_{n-1}^{\prime}$ and $\rho_{2}$ be such that $\rho_{1}<\rho_{2}<r_{\theta_{n-1}}$, close enough to $\rho_{1}$ so that for every $\rho^{\prime} \in\left[\rho_{1}, \rho_{2}\right]$,

$$
d_{F}\left(\phi_{\theta_{n-1}}\left(\rho^{\prime} z\right), \phi_{\theta_{n-1}}\left(\rho_{1} z\right)\right)<\frac{1}{2} \min \left(\eta, \varepsilon / 2^{n+1}\right)
$$

(this is possible since the injection of $C_{\varepsilon}^{\omega} \subset F$ is continuous for all $\varepsilon>0$ ). Let $\theta_{n}$ and $\rho_{n}^{\prime}$ be provided by Lemma 9 such that

- $\left|\theta_{n}-\theta_{n-1}\right|<\varepsilon / 2^{n+1}$,

- $\rho_{1}<\rho_{n}^{\prime}<\rho_{2}$,

- $r_{\theta_{n}}>\rho_{n}^{\prime}$,

- $d_{F}\left(\phi_{\theta_{n}}\left(\rho_{n}^{\prime} z\right), \phi_{\theta_{n-1}}\left(\rho_{n}^{\prime} z\right)\right)<\frac{1}{2} \min \left(\eta, \varepsilon / 2^{n+1}\right)$,

- $\phi_{\theta_{n}}\left(\rho_{n}^{\prime} z\right)$ does not belong to $K_{n} \cup D_{n} \cup L$.

We then define $\varepsilon_{n}=\frac{1}{2} d_{F}\left(K_{n} \cup D_{n} \cup L, \phi_{\theta_{n}}\left(\rho_{n}^{\prime} z\right)\right)$. It is easy to check that $H_{n}$ holds.

Now that the sequences $\theta_{n}, \rho_{n}^{\prime}, \varepsilon_{n}$ have been defined, let $\theta^{\prime}$ be the limit of the Cauchy sequence $\theta_{n}$, and $\rho^{\prime}$ the limit of the increasing sequence $\rho_{n}^{\prime}$ (which is bounded from above). Observe that for all $n, r_{\theta_{n}}>\rho_{n}^{\prime}$, and that by equation $(*)$ the sequence of maps $\phi_{\theta_{n}}\left(\rho_{n}^{\prime} z\right)$ (restricted to $\mathbb{D}$ ) is a Cauchy sequence for $d_{F}$, thus converges in $F$ to some $\psi$ (we use here the completeness of Fréchet spaces). Convergence in $F$ implies convergence in $C^{0}$; thus we can apply Lemma 4 to conclude 
that $\psi(z)=\phi_{\theta^{\prime}}\left(\rho^{\prime} z\right)$ on $\overline{\mathbb{D}}$. Also, $d_{F}\left(K_{n} \cup D_{n} \cup L, \psi\right) \geq \varepsilon_{n}$, so $\psi$ does not belong to any $K_{n}$ nor to any $D_{n}$. Since $\bigcup D_{n}=C^{\omega}$, this implies $\psi$ does not extend holomorphically to a neighborhood of $\overline{\mathbb{D}}$, thus $r_{\theta^{\prime}}=\rho^{\prime}$.

\section{Concluding Remark}

All of the above discussion extends to any analytic family for which an analogue of Lemma 2 can be proved.

\section{ACKNOWLEDGMENTS}

We would like to thank Adrien Douady for useful discussions. We would like to thank the referee for his constructive comments and his work in examining our paper.

\section{REFERENCES}

[A] A. Avila, Smooth Siegel disks via semicontinuity: A remark on a proof of Buff and Cheritat, math.DS/0305272

[ABC] A. Avila, X. Buff \& A. ChÉritat, Siegel disks with smooth boundaries, Acta Mathematica 193, 1-30 (2004). MR2155030 (2006e:37073)

[BC] X. Buff \& A. ChÉRItAT, Quadratic Siegel disks with smooth boundaries, preprint no. 242 at Université Paul Sabatier, Toulouse, France (2002).

[G] L. Geyer Smooth Siegel discs without number theory: A remark on a proof by Buff and Chéritat, submitted (2003).

[H1] M.R. HeRman, Sur la conjugaison différentiable des difféomorphismes du cercle à des rotations, Publ. Math. I.H.E.S. 49, 5-233 (1979). MR0538680 (81h:58039)

[H2] M.R. Herman, Are there critical points on the boundaries of singular domains?, Comm. Math. Phys. 99, 593-612 (1985). MR0796014 (86j:58067)

[M] J. Milnor, Dynamics in one complex variable, Introductory Lectures, Friedr. Vieweg \& Sohn, Braunschweig (1999). MR1721240(2002i:37057)

[PM1] R. PÉrez-Marco, Siegel disks with smooth boundary, Preprint (1997).

[PM2] R. PÉrez-Marco, Siegel disks with quasi-analytic boundary, Preprint no. 52 (1997) at Université Paris-Sud.

[Po] C. Pommerenke, Boundary Behavior of Conformal Maps, Grundlehren der mathematischen Wissenschaften 299, Springer-Verlag (1992). MR1217706 (95b:30008)

[PZ] C.L. Petersen \& S. Zakeri, On the Julia Set of a Typical Quadratic Polynomial with a Siegel disk, Ann. of Math. (2) 159 (2004), no. 1, 1-52. MR2051390 (2005c:37085)

[Ro] B. Rodin, Intrinsic rotations of simply connected regions, Complex Variables Theory Appl. 2 (1984), no. 3-4, 319-326. MR0743955 (85m:30026)

[Ru] W. Rudin, Functional Analysis, McGraw-Hill Series in Higher Math., second edition (1991). MR1157815 (92k:46001)

Université Paul Sabatier, Laboratoire Emile Picard, 118, route de Narbonne, 31062 Toulouse Cedex, France

E-mail address: buff@picard.ups-tlse.fr

Université Paul Sabatier, Laboratoire Emile Picard, 118, route de Narbonne, 31062 Toulouse Cedex, France

E-mail address: cheritat@picard.ups-tlse.fr 